\title{
Correlation of Amplitude-Integrated Electroencephalogram (aEEG) and Early Neurological Outcome in Newborn Treated with Therapeutic Hypothermia for Hypoxic Ischemic Encephalopathy (HIE): An Audit
}

\author{
Anitha Ananthan*, Ruchi Nanavati, Madhusudhan Shivamallappa and Abhishek Agrawal \\ Department of Neonatology, Seth GS Medical College and King Edward Memorial Hospital, Mumbai, India \\ *Corresponding Author: Anitha Ananthan, Department of Neonatology, Seth GS Medical College and King Edward Memorial Hospital, \\ Mumbai, India.
}

Received: July 26, 2019; Published: September 10, 2019

DOI: 10.31080/ASPE.2019.02.0143

\begin{abstract}
Aim: Amplitude-integrated electroencephalogram (aEEG) is a commonly used monitoring tool for assessing cortical electrical activity and can also detect seizures. The aim of the study was to correlate amplitude integrated electroencephalogram (aEEG) with short term neurological outcomes in term neonates post therapeutic hypothermia for HIE.

Methods: This was a retrospective observational study conducted in level III NICU in a tertiary care hospital. Neonates ( $\mathrm{N}=40)$ treated with therapeutic hypothermia for HIE and who were monitored with aEEG during 2 year period were included in the audit. The details of complete neurological examination at discharge and subsequent motor assessment using INFANIB scoring tool on follow up were recorded. The documented aEEG readings of these neonates were scored for the back ground pattern, sleep - wake cycle and seizures. The aEEG findings were correlated with neurological examination using scoring system.

Results: Among 9/40 infants who had normal neurological exam, 55.5\% had aEEG score $\leq 4$ and remaining $44.5 \%$ had score between 5 - 8. Among 17/40 infants who had transient neurological assessment score, $17.64 \%$ had aEEG score $\leq 4$ and remaining $82.35 \%$ had score between 5 to 8. Among 14/40 infants who had abnormal neurological exam, 78.5\% had score between 5 to 8 and remaining $21.42 \%$ had score $\geq 8$. aEEG findings were correlated with neurological examination scoring using Spearman's rank correlation coefficient (rs value $0.6075, \mathrm{P}=0.001$ ) and found to have moderate positive correlation.

Conclusion: aEEG monitoring in infants with moderate to severe HIE correlates significantly with short term neurological outcomes. Keywords: Amplitude Integrated Electroencephalogram; Hypoxic Ischemic Encephalopathy; Neonate; Therapeutic Hypothermia
\end{abstract}

\section{Key Notes}

- Amplitude-integrated electroencephalogram (aEEG) is a commonly used monitoring tool for assessing cortical electrical activity and can also detect seizures.

- $\quad$ aEEG monitoring in infants with moderate to severe HIE correlates significantly with short term neurological outcomes

- The timely detection of short term neurological abnormalities with the aid of aEEG enables timely initiation of early intervention therapy. This may help to achieve better long term neurological outcome.

\section{Abbreviations}

aEEG: Amplitude Integrated Electroencephalogram; CFM: Cerebral Function Monitor; HIE: Hypoxic Ischaemic Encephalopathy;
INFANIB: Infant Neurological International Battery; MRI: Magnetic Resonance Imaging; NICU: Neonatal Intensive Care Unit; SE: Status Epilepticus; SWC: Sleep Wake Cycle; TH: Therapeutic Hypothermia

\section{Introduction}

Intrapartum hypoxic events are a major cause of neonatal mortality responsible for approximately one in five of all neonatal deaths worldwide, causing an estimated 717,000 deaths in 2010 [1]. Therapeutic hypothermia (TH) is one of the proven therapy that improves the neurological outcome of neonates with HIE [2].

TH is a modality of treatment in which the core temperature of the vulnerable deep brain structures, the basal ganglia, is reduced to 33 - 34 degrees Celsius for 72 hrs to prevent neuronal injury [3]. Hypothermia reduces cerebral oxygen consumption, inhibits syn- 
Correlation of Amplitude-Integrated Electroencephalogram (aEEG) and Early Neurological Outcome in Newborn Treated with Therapeutic Hypothermia for Hypoxic Ischemic Encephalopathy (HIE): An Audit

thesis, release, and/or reuptake of ischemia-induced neurotransmitters and neuromodulators. Mild to moderate hypothermia inhibits the release of reactive oxygen species including hydrogen peroxide $[4,5]$ and prevent activation of apoptotic mechanisms. Even a small reduction in temperature provides significant neuroprotection after ischemic neuronal injury $[3,6]$.

Amplitude-integrated electroencephalogram (aEEG) is a commonly used monitoring tool for assessing cortical electrical activity and can also detect seizures [7]. In neonates with moderate or severe HIE, monitoring for the presence of electrographic seizures is recommended as it provide prognostic information [7]. An abnormal aEEG background pattern has been shown to correlate with an abnormal MRI in uncooled patients with HIE [8].

The association between aEEG and long-term neurological outcome has been extensively studied and proven. We could find only a few studies from India which has studied the role of therapeutic hypothermia on HIE [9-12]. To our knowledge this is the pioneer study in India which has correlated neurological outcome of neonates with HIE who received TH and aEEG.

The aim of the study was to correlate aEEG with short term neurological outcomes in term neonates post therapeutic hypothermia for HIE.

\section{Methods}

A retrospective Observational study was conducted after approval by the Institutional Ethics Committee at level III NICU in a tertiary care hospital. Neonates who were treated with therapeutic hypothermia for HIE and monitored with aEEG during a period of 2 years from january 2016 till February 2018 were included in the study. Therapeutic Hypothermia was provided to infants with gestation $\geq 37$ weeks, birth weight $>1,800 \mathrm{~g}$, having evidence of physiologic compromise with any 1 of $4: 1$ ) History of an acute perinatal event (abruptio placenta, cord prolapse, severe FHR abnormality, e.g. variable or late decelerations) 2) An Apgar score $<5$ at 10 minutes 3) Continued need for ventilation initiated at birth and continued for at least 10 minutes 4 ) Cord $\mathrm{pH}$ or first postnatal blood gas $\mathrm{pH}$ at $<1$ hour $<=7.0$ or base deficit $>=16 \mathrm{mEq} / \mathrm{L}$ and moderate or severe HIE diagnosed on clinical examination.

\section{Sample size}

Since this was a retrospective study and a pilot project, we analysed the aEEG pattern of 40 neonates during the time period 2016 - 2018 who received TH for moderate to severe HIE Data was collected from the therapeutic hypothermia record book maintained in the NICU. Hypothermia was provided by a machine named Techo therm neo. Temperature of the term baby who fulfilled the criteria for TH was maintained between $33.5-34.5^{\circ} \mathrm{C}$ for 72 hours. This was followed by rewarming phase which was done over $6 \mathrm{hrs}$. Neonates who received therapeutic hypothermia were monitored with aEEG using Natus Olympus Brainz machine. Demographic data of the patient, duration of stay in hospital and outcomes of the study were recorded. The Clinical HIE grading (classified using Sarnath and Sarnath classification) was recorded. Details of complete neurological examination at discharge and subsequent motor assessment using INFANIB [13] tool on follow up were recorded. These patients were grouped in 3 categories: 1) abnormal $(<48) 2$ 2) transient (49 - 65) 3) normal ( $\geq 66$ ) according to their assessment in INFANIB scale [13].

The aEEG data was retrieved from the archived memory of the CFM monitor and was anonymized, coded and confidential information was submitted for one of the faculty in the department for review. aEEG was analyzed for following parameters.

\section{Back ground pattern}

1. Continuous normal voltage: continuous activity with lower (minimum) amplitude around $>5 \mu \mathrm{V}$ and maximum amplitude $>10 \mu \mathrm{V}$.

2. Discontinuous: Discontinuous background with minimum amplitude variable, but below $5 \mathrm{mcV}$, and maximum amplitude above $10 \mathrm{mcV}$.

3. Burst-Suppression: discontinuous background with minimum amplitude without variability at $0-1 \mu \mathrm{V}$, and bursts with amplitude $>25 \mu \mathrm{V}$.

4. BS+ means burst density $\geq 100$ bursts/h, and BS- means burst density $<100$ bursts $/ \mathrm{h}$.

5. Continuous low voltage: continuous background pattern of very low voltage (around or below $5 \mu \mathrm{V}$ )

6. Inactive, flat: Mainly inactive (isoelectric tracing) background below $3-5 \mu \mathrm{V}$.

Sleep-wake cycling (SWC): SWC in the aEEG is characterized by smooth sinusoidal variations, mainly in the minimum amplitude.

1. No SWC: No cyclic variation of the aEEG background;

2. Imminent/immature SWC: Some, but not fully developed, cyclic variation of the lower amplitude, but not developed as compared to normative gestational age representative data;

3. Developed SWC: Clearly identifiable sinusoidal variations between discontinuous and more continuous background activity.

\section{Seizures}

Single seizure; (II) repetitive seizures ( $\geq 3$ seizure patterns during a 30-minute period); and (III) status epilepticus (SE) continu- 
Correlation of Amplitude-Integrated Electroencephalogram (aEEG) and Early Neurological Outcome in Newborn Treated with Therapeutic Hypothermia for Hypoxic Ischemic Encephalopathy (HIE): An Audit

ous seizure pattern for $\geq 30$ minutes, presenting as a "saw tooth pattern" or as continuous increases of the lower and upper margins [14].

The cerebral function monitoring scores were classified in three grades as Grade I ( $\leq 4)$, Grade II $(>4-$ to $\leq 8)$ and Grade III $(>8)$ [15-17].

\section{Statistical analysis}

Statistical analysis was performed using SPSS software. To assess the strength of the association between EEG findings (graded from 1 to 3 ) and outcome at follow-up (graded from 1 to 3), Spearman's rank correlation coefficient was used and data was analyzed.

\section{Results}

We retrospectively analyzed aEEG of 40 infants with grade II and grade III HIE who were treated with therapeutic hypothermia in our NICU during the past 2 yrs (2016 to 2018). We analyzed aEEG findings in the included infants and scored using aEEG scoring system devised by Luo., et al. This was correlated with the neurological outcome at 3 months of corrected age by INFANIB scale.

The infant characteristics of the study population is given in table 1 . We could find that $77.5 \%$ of the study population had stage 2 HIE and remaining $32.5 \%$ had stage 3 HIE. aEEG background pattern was normal in 5\% of the study population. Abnormal aEEG pattern was found in rest $95 \%$ of the study population which constituted discontinuous pattern and burst suppression pattern in $47.5 \%$ each of the study group. As per the aEEG scoring system, $20 \%$ of the study population had score $\leq 4,72.5 \%$ had score between 4 to 8 and rest $7.5 \%$ of the study population had score $\geq 8$. Sleep wake cycle was present in only $17.5 \%$ of the study group.

\begin{tabular}{|c|c|c|c|c|}
\hline Serial no & & & $\mathbf{N}$ & Percentage (\%) \\
\hline \multirow{2}{*}{1} & \multirow{2}{*}{ Birth weight } & $<2.5 \mathrm{~kg}$ & 8 & 20 \\
\hline & & $>2.5 \mathrm{~kg}$ & 32 & 80 \\
\hline \multirow{2}{*}{2} & \multirow{2}{*}{ Apgar score } & $<3$ at 5 min & 13 & 32.5 \\
\hline & & $>3$ at $5 \mathrm{~min}$ & 27 & 67.5 \\
\hline \multirow{2}{*}{3} & \multirow{2}{*}{ sex } & male & 24 & 60 \\
\hline & & female & 16 & 40 \\
\hline \multirow{2}{*}{4} & \multirow{2}{*}{ HIE } & Grade 2 & 31 & 77.5 \\
\hline & & Grade 3 & 9 & 32.5 \\
\hline \multirow{2}{*}{5} & \multirow{2}{*}{ Seizure } & present & 26 & 65 \\
\hline & & absent & 14 & 35 \\
\hline \multirow{3}{*}{6} & \multirow{3}{*}{$\begin{array}{l}\text { Neurological } \\
\text { abnormality } \\
\text { at } 3 \text { month }\end{array}$} & abnormal & 14 & 35.0 \\
\hline & & transient & 17 & 42.5 \\
\hline & & normal & 9 & 22.5 \\
\hline
\end{tabular}

Table 1: Showing the infants characteristics.
We followed up the study participants post discharge from the NICU and neurological examination was performed at 3 months of corrected age using INFANIB scale. Normal neurological examination was observed in $9 / 40$ (22.5\%) study participants, transient abnormality was found in 17/40 (42.5\%) and abnormal neurological exam was found in $14 / 40$ (35\%) of the study population respectively.

We analyse infants as per their neurological outcomes with aEEG scores which is shown in table 2. Among 9/40 infants who had normal neurological exam, 55.5\% had aEEG score $\leq 4$ and remaining 44.5\% had score between 5 - 8. Among 17/40 infants who had transient neurological assessment score, $17.64 \%$ had aEEG score $\leq 4$ and remaining $82.35 \%$ had score between 5 to 8 . Among $14 / 40$ infants who had abnormal neurological exam, 78.5\% had score between 5 to 8 and remaining $21.42 \%$ had score $\geq 8$.

\begin{tabular}{|c|c|c|c|}
\hline $\begin{array}{c}\text { Neurological } \\
\text { outcome (N, \%) }\end{array}$ & aEEG (N) (\%) & $\mathbf{N}$ & Percentage (\%) \\
\hline \multirow{3}{*}{$\begin{array}{l}\text { Normal } \\
(\mathrm{N}=9,22.4 \%))\end{array}$} & $\leq 4$ & 5 & 55.5 \\
\hline & $5-8$ & 4 & 44.5 \\
\hline & $>8$ & 0 & 0 \\
\hline \multirow{3}{*}{$\begin{array}{l}\text { Transient } \\
(\mathrm{N}=17,42.5 \%)\end{array}$} & $\leq 4$ & 3 & 17.64 \\
\hline & $5-8$ & 14 & 82.35 \\
\hline & $>8$ & 0 & 0 \\
\hline \multirow{3}{*}{$\begin{array}{l}\text { Abnormal } \\
(\mathrm{N}=14,35 \%)\end{array}$} & $\leq 4$ & 0 & 0 \\
\hline & $5-8$ & 11 & 78.5 \\
\hline & $>8$ & 3 & 21.42 \\
\hline \multicolumn{4}{|l|}{ HIE stage } \\
\hline \multirow{3}{*}{$\begin{array}{l}\text { Stage II } \\
(\mathrm{N}=31,77.5 \%)\end{array}$} & $\leq 4$ & 8 & 25.8 \\
\hline & $5-8$ & 21 & 53.84 \\
\hline & $>8$ & 2 & 6.45 \\
\hline \multirow{3}{*}{$\begin{array}{l}\text { Stage III } \\
(\mathrm{N}=9,22.5 \%)\end{array}$} & $\leq 4$ & 0 & 0 \\
\hline & 5-8 & 8 & 88.88 \\
\hline & $>8$ & 1 & 11.11 \\
\hline
\end{tabular}

Table 2: aEEG and neurological outcome.

We further analysed the neurological examination findings as per the HIE staging (Table 3). In those infants with stage 2 HIE, $25.58 \%$ had normal, $48.38 \%$ had transient and $25.58 \%$ respectively had abnormal neurological findings at 3 months follow up. In those infants with stage $3 \mathrm{HIE}, 11.11 \%$ had normal, $22.22 \%$ had transient and $66.66 \%$ had abnormal neurological findings respectively at 3 months follow up.

With these findings, we correlated the aEEG findings with neurological examination scoring using Spearman's rank correlation 
Correlation of Amplitude-Integrated Electroencephalogram (aEEG) and Early Neurological Outcome in Newborn Treated with Therapeutic Hypothermia for Hypoxic Ischemic Encephalopathy (HIE): An Audit

\begin{tabular}{|l|l|l|c|}
\hline \multirow{2}{*}{ HIE stage } & $\begin{array}{l}\text { Neurological outcome at } \\
\text { 3 months using INFANIB }\end{array}$ & $\mathbf{N}$ & $\mathbf{\%}$ \\
\hline \multirow{3}{*}{ Stage II } & Normal & 8 & 25.58 \\
\cline { 2 - 4 } & Transient & 15 & 48.38 \\
\cline { 2 - 4 } & Abnormal & 8 & 25.58 \\
\hline \multirow{3}{*}{ Stage III } & Normal & 1 & 11.11 \\
\cline { 2 - 4 } & Transient & 2 & 22.22 \\
\cline { 2 - 4 } & Abnormal & 6 & 66.66 \\
\hline
\end{tabular}

Table 3: HIE stage and neurological outcome.

\section{Discussion}

Perinatal asphyxia is one of the leading cause of neonatal mortality all over the world. This retrospective study was undertaken to correlate aEEG in infants with moderate to severe HIE with their neurological findings at 3 months of age. Though the results obtained in the study showed that aEEG was abnormal in majority of the study participants, severe aEEG abnormality was found in only in $7.5 \%$ of the study group. These findings were correlated with short term neurological outcomes which showed significant correlation.

The correlation between aEEG and short term neurological outcome at 3 and 6 hour s of life was studied by Toet., et al [18]. The study found that sensitivity and specificity for burst suppression pattern together with flat tracing and continuous low voltage tracing for poor outcome at 3 hours was 0.85 and 0.77 , respectively; at 6 hours 0.91 and 0.86 respectively. The positive predictive value was $78 \%$ and negative predictive value was $84 \%$ at 3 hours after birth. At 6 hours, positive predictive value was $86 \%$ and negative predictive value was $91 \%$. The study concluded that aEEG could be very useful for selecting infants who could be benefitted from intervention after birth asphyxia. A close correlation between the aEEG and subsequent neurological outcome at 18 to 24 months of age was found in study by Naqeeb., et al [19].

The diagnostic value of aEEG in predicting outcome of newborn infants was done by Luo F., et al [17]. The neuromotor assessment was assessed by using INFANIB which was similar to our study. Chi square analysis and Spearman rank correlation analysis showed that aEEG pattern correlated with neurological outcomes of those infants. These findings were similar to our findings though they assessed long term neurological outcomes.

To our knowledge this is the first study in Indian scenario which tried to correlate aEEG patterns with short term neurological outcome at 3 month of corrected age. The study demonstrated that the aEEG was well correlated with the short term neurological outcomes in the asphyxiated neonates. This recommends the regu- lar use of aEEG monitoring and the scoring system for predicting abnormal neurological outcomes in the neonates who are getting treated for perinatal asphyxia. The limitation of our study was that it was retrospective analysis and lacked the power and clarity of prospective observational study or randomized controlled trial.

In neonates with perinatal asphyxia who presents with moderate to severe HIE, strict aEEG monitoring and scoring is warranted throughout the treatment period. This will help Neonatologists to ensure vigilant neurological examination post discharge, at 3, 6, 9 and 12 months of age of those infants with perinatal asphyxia. The correlation of aEEG with long term neurological examination findings need to be done so that that current findings can be extrapolated to the long term outcomes.

\section{Conclusion}

In view of the results obtained, it can be concluded that aEEG monitoring in infants with moderate to severe HIE correlates significantly with short term neurological outcomes. However, the findings need to be confirmed for long term neuromotor outcomes and neurodevelopmental outcomes. We recommend a properly designed observational study/RCT with adequate power targeting both short and long term neurological findings to confirm our findings.

\section{Acknowledgements}

Nil.

\section{Conflict of Interest}

The authors declare no conflict of interests.

Funding

Nil.

\section{Bibliography}

1. Liu L., et al. "Global, regional, and national causes of child mortality: an updated systematic analysis for 2010 with time trends since 2000". Lancet 379.9832 (2012): 2151-2161.

2. Rivera-Lara L., et al. "Therapeutic hypothermia for acute neurological injuries". Neurotherapeutics 9.1 (2012): 73-86.

3. Edwards AD., et al. "Neurological outcomes at 18 months of age after moderate hypothermia for perinatal hypoxic ischaemic encephalopathy: synthesis and meta-analysis of trial data". BMJ 340 (2010): c363.

4. Globus MY., et al. "Effect of ischemia on the in vivo release of striatal dopamine, glutamate, and gamma-aminobutyric acid studied by intracerebral microdialysis". Journal of Neurochemistry 51.5 (1988): 1455-1464. 
5. Okuda C., et al. "Alteration of the turnover of dopamine and 5-hydroxytryptamine in rat brain associated with hypothermia". Pharmacology Biochemistry and Behavior 24.1 (1986): 79-83.

6. Lei B., et al. "The effect of hypothermia on $\mathrm{H} 2 \mathrm{O} 2$ production during ischemia and reperfusion: a microdialysis study in the gerbil hippocampus". Neuroscience Letters 222.2 (1997): 9194.

7. Hasegawa M., et al. "Hypothermic inhibition of apoptotic pathways for combined neurotoxicity of iron and ascorbic acid in differentiated PC12 cells: reduction of oxidative stress and maintenance of the glutathione redox state". Brain Research 1283 (2009): 1-13.

8. Padden B., et al. "Does amplitude-integrated electroencephalogram background pattern correlate with cerebral injury in neonates with hypoxic-ischaemic encephalopathy?". Journal of Paediatrics and Child Health 51.2 (2015):180-185.

9. Lally PJ., et al. "Neonatal encephalopathic cerebral injury in South India assessed by perinatal magnetic resonance biomarkers and early childhood neurodevelopmental outcome". PloS one 9 (2014): e87874.

10. Jose A., et al. "Correlation of EEG, CT, and MRI Brain with Neurological Outcome at 12 Months in Term Newborns with Hypoxic Ischemic Encephalopathy". Journal of Clinical Neonatology 2.3 (2013): 125-130.

11. Bharadwaj SK and Bhat BV. "Therapeutic hypothermia using gel packs for term neonates with hypoxic ischaemic encephalopathy in resource-limited settings: a randomized controlled trial". Journal of Tropical Pediatrics 58.5 (2012):382-388.

12. Chandrasekaran M., et al. "Therapeutic Hypothermia for Neonatal Encephalopathy in Indian Neonatal Units: A Survey of National Practices". Indian Pediatr 54.11 (2017): 969-970.

13. Solimani F KE and Shams S. "Report of screening of infant (418) months in Karaj for delay development". Journal of Rehabilitation 6-7 (2001): 22-28.

14. Abend NS., et al. "EEG monitoring during therapeutic hypothermia in neonates, children, and adults". American Journal of Electroneurodiagnostic Technology 51.3 (2011): 141-164.

15. Hellstrom-Westas L and Rosen I. "Continuous brain-function monitoring: state of the art in clinical practice". Seminars in Fetal and Neonatal Medicine 11.6 (2006): 503-511.
16. Lena Hellström-Westas Linda dV. "Amplitude-integrated EEG Classification and Interpretation in Preterm and Term Infants". Neo Reviews (2006)

17. Luo F., et al. "Amplitude-integrated electroencephalographic changes in neonates with acute bilirubin encephalopathy". Zhonghua Er Ke Za Zhi 51.3 (2013): 221-226.

18. Toet MC., et al. "Amplitude integrated EEG 3 and 6 hours after birth in full term neonates with hypoxic-ischaemic encephalopathy". Archives of Disease in Childhood Fetal Neonatal 81.1 (1999): F19-F23.

19. Al Naqeeb N., et al. "Assessment of neonatal encephalopathy by amplitude-integrated electroencephalography". Pediatrics 103.6-1 (1999): 1263-1271.

Volume 2 Issue 10 October 2019

(C) All rights are reserved by Anitha Ananthan., et al. 